\title{
Development of a Thin-Film Stretchable Electrical Interconnection Technology for Biocompatible Applications
}

\author{
R. Verplancke*, T. Sterken, F. Axisa, J. Vanfleteren \\ Centre for Microsystems Technology (CMST)/IMEC, ELIS Department, Ghent University, Technologiepark 914a, \\ 9052 Zwijnaarde, Belgium \\ *E-mail: Rik.Verplancke@UGent.be \\ Tel: +3292645351
}

\begin{abstract}
Stretchable electronics technologies have gained a lot of interest for reasons such as user comfort and reliability. Key aspect in these technologies is the fabrication of stretchable electrical interconnections. These are realized by patterning an intrinsic, non-stretchable gold film into a sequence of horseshoe shapes, acting as "2D" springs when embedded into PDMS. Polyimide is used as a supporting material, successfully enhancing reliability during mechanical loading. This was illustrated by application of various cyclic uni-axial strains to test structures which were fabricated in this technology. A lifetime over 130 '000 and 500'000 cycles has been shown at strains of respectively $20 \%$ and $10 \%$.
\end{abstract}

\section{Introduction}

Stretchable electronics technologies have gained a lot of interest in applications where the ability of electronic circuits to deform is an advantage or even a necessity. Biomedical devices in particular take advantage of these emerging technologies by improved user comfort, enhanced reliability and realization of a soft interface between device and tissue, again improving comfort. Furthermore, stretchable microelectrode arrays allow studying cellular activity when cells are subjected to mechanical strains.

The fabrication of stretchable electrical interconnections is a key aspect for the realization of stretchable electronics. The interconnections should allow for electrical conduction at an application-induced strain, and ensure this conduction during long term use. Different approaches are found in literature ranging from the use of intrinsically stretchable conductive materials to out-ofplane or in-plane patterning of intrinsically nonstretchable conductors to cope with applied strains. In one approach [1], straight tracks of a very thin gold film $(50 \mathrm{~nm})$ are evaporated on an elastomeric polymer (i.e. PDMS). A matrix of micro-cracks is introduced in the gold film which behaves like an accordion upon application of strain: cracks open and close when stretched.

In this contribution, a new technology for the fabrication of stretchable electrical interconnections based on the in-plane patterning of a metal film is presented. Special attention is given to improve reliability under cyclic loading, but also to possible use in biomedical applications.

\section{Stretchable Electronics Concept}

As electronic components are typically rigid or flexible, the main challenge in the development of stretchable electronics technologies consists in the fabrication of electrical interconnections which are still conductive during and after stretching. These can then be used as a means to interconnect rigid or flexible islands of components. A stretchable circuit thus typically consists of multiple functional islands, connected by stretchable interconnections.

The approach described in this paper focuses on inplane patterning of a metal film into a sequence of meandering shapes. Finite element analysis, described in detail elsewhere [2], was used to determine the optimal design. The resulting basic repetitive unit, the horseshoe shape, together with its design parameters (angle $\alpha$, width $w$ and radius $r$ ) is depicted in Figure 1. By embedding meandering tracks in a subsequent step into an elastomeric polymer such as PDMS, these are able to act as "2D" springs.

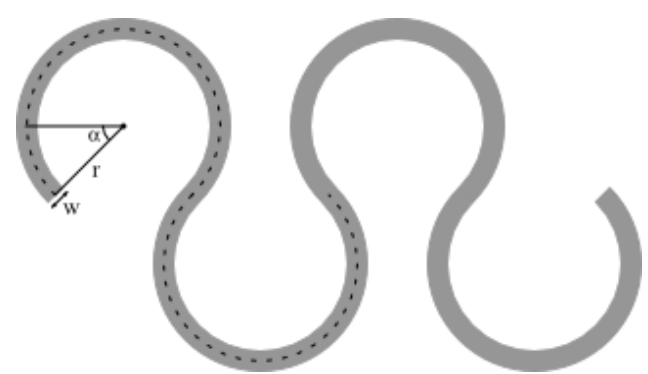

Figure 1: Stretchable electrical interconnections are realized using a sequence of horseshoe shapes; these are defined by the angle $\alpha$, width $w$ and radius $r$.

It was found that the reliability of stretchable interconnections under cyclic loading is limited by crack formation. To experimentally verify the different failure modes, interconnections were fabricated out of a $9 \mu \mathrm{m}$ copper sheet (RA copper) laminated on top of a $25 \mu \mathrm{m}$ polyimide foil (purchased from UBE). The technology which is used to realise the test vehicles is described in [3]. These interconnections were subjected to a cyclic uniaxial strain and visually inspected by means of an optical microscope afterwards.

Two failure modes were experimentally observed: accumulation of plastic deformation (inner side of the left track in Figure 2 - indicated by purple rectangle) and 
buckling (outer side of the left track and inner side of the right track in Figure 2 - indicated by blue rectangle).

The accumulation of plastic deformation will eventually lead to crack formation and propagation. The formation of the crack is delayed if the width of the track is reduced, as the maximum plastic strain decreases. The propagation of the crack is reduced by using a (meandering) supporting polymer material with higher Young's modulus than PDMS, such as polyimide.

Polyimide PI-2611 (HD Microsystems) [4] was chosen as a supporting material for the stretchable electrical interconnections. PI-2611 films exhibit desirable properties such as high modulus $(8.5 \mathrm{GPa})$, low moisture uptake $(0.5 \%)$, low stress and low CTE $\left(3 \mathrm{ppm}{ }^{\circ} \mathrm{C}^{-1}\right)$, making it ideally suited for various thin-film deposition steps.
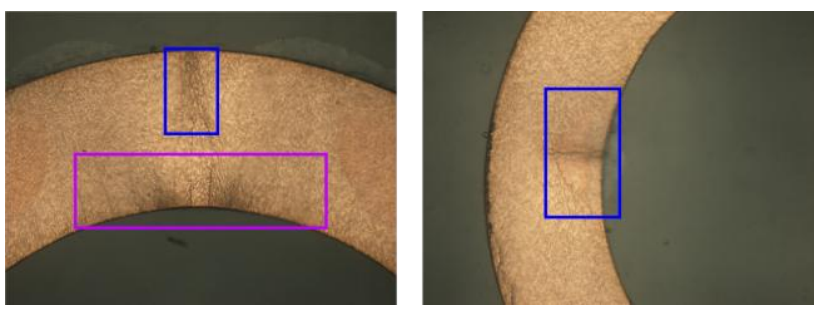

Figure 2: Crack formation in horseshoe-shaped interconnections.

The second failure mode, buckling, results in an accumulation of plastic strain on the top surface of the copper layer, eventually leading to crack propagation. The plastic deformation due to buckling is reduced by using thin-film metallization. The buckling of the meander can be avoided by decreasing the width of the track. Note that decreasing the width of the meander is beneficial for avoiding both buckling and strain accumulation failure mechanisms.

\section{Fabrication Process}

A schematic representation of the fabrication process is shown in Figure 3, and is briefly discussed hereafter.

Fabrication of the horseshoe shapes is done on a rigid carrier before being embedded into PDMS. At this point, a rigid carrier on which a release stack is deposited serves as a starting point for the fabrication process. The release stack will be discussed at the end of this section.

In a first step, $5 \mu \mathrm{m}$ polyimide PI-2611 is spin-coated (3000rpm for $30 \mathrm{~s})$ and cured $\left(350^{\circ} \mathrm{C}\right)$ on this base substrate. Next, a 50nm thick titanium-tungsten adhesion layer and a 200nm thick gold film is sputter deposited. Both metal layers are patterned (horseshoe shapes) using photolithography. For this purpose, positive photoresist S1818 $(4000 \mathrm{rpm} ; 1.8 \mu \mathrm{m})$ is used. After photoresist strip in acetone, another $5 \mu \mathrm{m}$ layer of polyimide PI-2611 is spin-coated and cured.

To pattern both the supporting bottom and top polyimide layer, an additional hard mask is required; a $500 \mathrm{~nm}$ thick aluminum film is sputter deposited and patterned using photolithography based on positive photoresist S1818 (4000rpm; 1.8 $\mu \mathrm{m})$. Next, dry etching (RIE) of polyimide is performed using a System VII Batchtop RIE system (Plasma-Therm). The etch recipe was optimized with respect to etch rate and uniformity over the surface. Optimal etching conditions were found with a vacuum pressure of $150 \mathrm{mT}$ Trr, gas flow rates $\mathrm{O}_{2}: \mathrm{CHF}_{3}$ of $15: 5 \mathrm{sccm}$ and $150 \mathrm{~W}$ RF power. These parameters result in an etch rate of approximately $0.33 \mu \mathrm{m}$ $\min ^{-1}$. The aluminum hard mask is finally etched, leaving polyimide supported electrical interconnections on top of the release stack.

(a) Spin-coat PI-2611 on rigid carrier with release stack

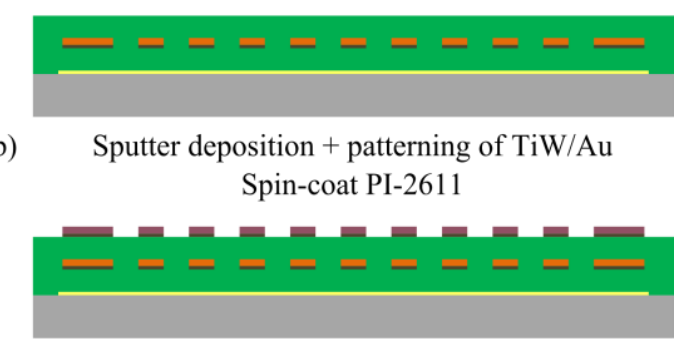

(c) Sputter deposition + patterning of Al hard mask

(d)

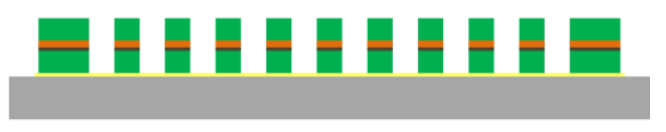

Dry etch PI-2611

Strip hard mask

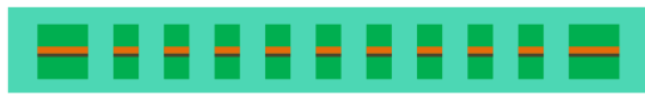

(e)

Embed into PDMS (by moulding, ...)

Figure 3: Schematic representation of the fabrication process.

At this point, the structured films can be embedded into PDMS. This is done by liquid injection moulding in two phases. Sylgard 186 (Dow Corning) is prepared by mixing base and curing agent in a 10:1 mixing ratio by weight and degassed to remove air bubbles. 1200 OS Primer (Dow Corning) is applied on the substrate to improve adhesion between PDMS and polyimide. The substrate is subsequently clamped in between 2 PMMA moulds and Sylgard 186 is injected and cured at $50^{\circ} \mathrm{C}$ for 4 hours. This step is illustrated in Figure 4 (left).
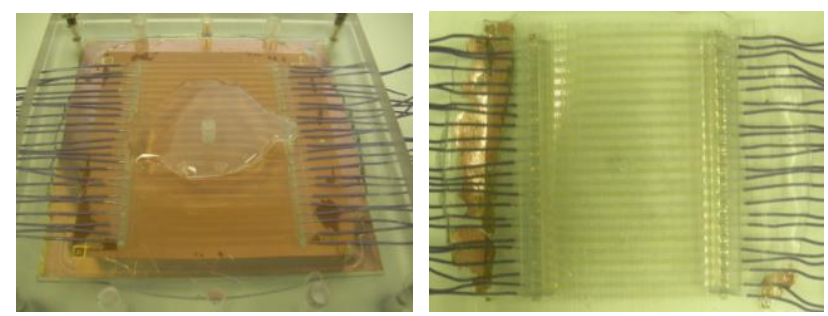

Figure 4: Moulding into PDMS by use of PMMA moulds (left) and moulded sample (right). 
To embed the second side, the stack has to be released from its rigid carrier. Due to the variety of processing conditions of the process flow discussed above, an appropriate release technique had to be developed. The release technique should be compatible with the hightemperature curing scheme (up to $350^{\circ} \mathrm{C}$ ) of polyimide PI2611 and all of the steps required for pattern definition of the functional stack. In addition, it should also allow an easy release after the first moulding step.

Up to now, a single release layer complying with these requirements has not been found. Therefore, a release technique based on the use of $\mathrm{KCl}$ was developed. A $500 \mathrm{~nm}$ thick layer is selectively thermally evaporated; a shadow mask is used to leave the edges of the rigid carrier open. An additional protective layer is needed to shield the salt against all of the subsequent processing steps. To this end, a $1 \mu \mathrm{m}$ copper and a $50 \mathrm{~nm}$ TiW film was sputter deposited.

Release of the stack can thus be done by unmolding the substrate and incising the upper PDMS layer. By immersing the substrate into deionised water, the release layer is dissolved and the stack can be successfully released. The protective films are etched, 1200 OS Primer is applied and a moulding step identical to the first one is repeated. The moulded result can be seen in Figure 4 (right); a close-up can be seen in Figure 5.

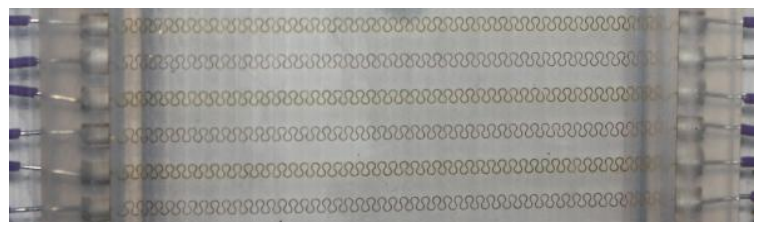

Figure 5: Horseshoe-shaped interconnections embedded into PDMS.

\section{Results}

Test structures consisting of straight horseshoe-shaped tracks of approximately $45 \mathrm{~mm}$ were fabricated in this technology (Figure 5). The design parameters (indicated in Figure 1) were fixed at an angle $\alpha=45^{\circ}$, radius $r=350 \mu \mathrm{m}$ and track width $w=100 \mu \mathrm{m}$. Two widths of polyimide support are available: a width of $100 \mu \mathrm{m}$ (same width as gold track) and of $200 \mu \mathrm{m}(100 \mu \mathrm{m}$ wider than gold track) are designed on the same mask. A close-up of polyimide supported horseshoe shapes embedded into PDMS on one side can be seen in Figure 6.

Electrical characterization of interconnections during mechanical tests is done with an in-house built ohmmeter. To this end, before embedding into PDMS, contact pads are opened simultaneously with the structuring of polyimide. Hereby, gold pads serve as a stop layer for the dry etching. Electrical wires are glued on these pads by means of an isotropic conductive adhesive (CE 3103, Emerson \& Cuming), providing a reliable electrical contact. In a next step, moulding is performed as described in the fabrication process. This resulted in a final thickness of $2.86 \mathrm{~mm}$.
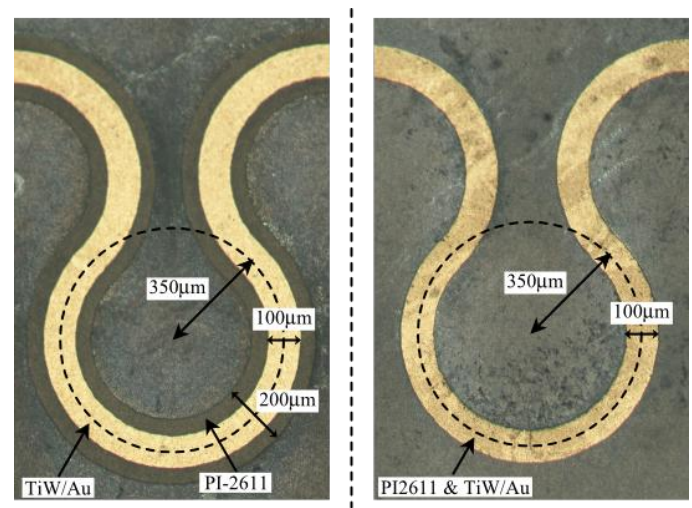

Figure 6: Polyimide supported horseshoe shapes embedded into PDMS on one side, also illustrating the design of the test structures for mechanical tests.

Mechanical tests are performed using an Instron 5543 (Figure 7). The latter allows for automated application of cyclic uni-axial strain and collection of data (resistance versus strain and time). The tests are typically performed on four tracks simultaneously; moulded samples are thus cut accordingly using a knife.

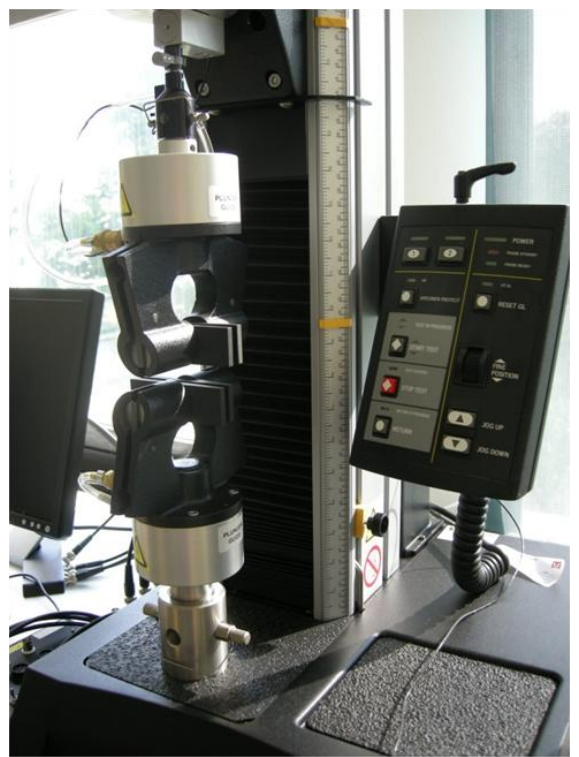

Figure 7: Instron 5543 used for mechanical tests.

Prior to stretching, the resistance of the tracks was measured and served as a reference during mechanical tests. The initial value was approximately $156 \Omega$. Considering a path length of the tracks of approximately $16.7 \mathrm{~cm}$, this value is in line with the theoretical expected resistance. Any discrepancies can be explained by a small deviation of the gold film thickness from its desired thickness (200nm).

Several strains were applied to the test structures. In a first test, the test structures were subjected to a one-time uni-axial strain and were found to be conductive up to strains of 100-110\%. Next, different cyclic uni-axial strains were applied at a constant strain rate of $10 \% \mathrm{sec}^{-1}$. The results of these tests are summarized in Table 1 (and 
graphically represented in Figure 8) and shortly discussed hereafter.

\begin{tabular}{|c|c|c|}
\hline $\begin{array}{c}\text { Strain } \\
{[\%]}\end{array}$ & $\begin{array}{c}\text { PI support } \\
100 \mu \mathrm{m}\end{array}$ & $\begin{array}{c}\text { PI support } \\
200 \mu \mathrm{m}\end{array}$ \\
\hline 60 & 32 & $8^{\prime} 220^{*}$ \\
\hline 40 & 216 & $37^{\prime} 000^{*}$ \\
\hline 20 & $44^{\prime} 900$ & $>130^{\prime} 000$ \\
\hline 10 & $>500^{\prime} 000$ & $>500^{\prime} 000$ \\
\hline
\end{tabular}

: Elucidated in the text

Table 1: Average number of cycles before electrical failure of test structures when subjected to cyclic uniaxial strain.

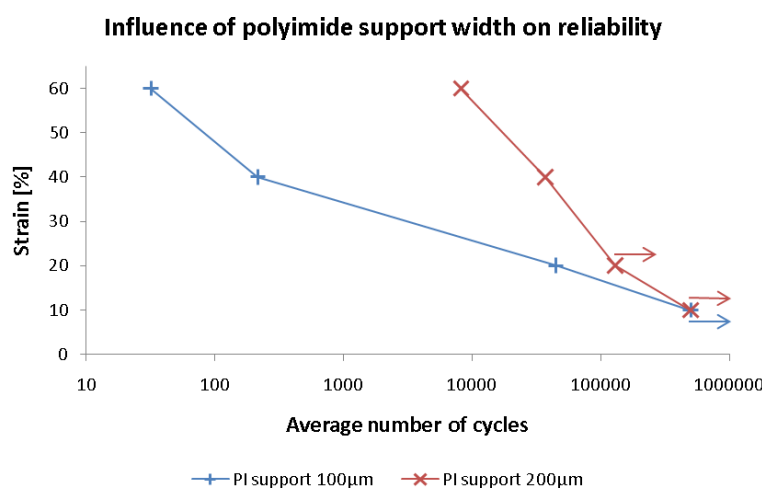

Figure 8: Graphical representation of Table 1.

At the higher strains, the test structures with a polyimide support of $100 \mu \mathrm{m}$ width led to earlier failure than those with a $200 \mu \mathrm{m}$ wide polyimide support. It has to be noted that during the cyclic loading at strains of $40 \%$ and $60 \%$, the PDMS started tearing (see Figure 9) at an average of respectively $37^{\prime} 000$ and 8'220 cycles due to the manual cutting of the test structures where side cuts in the PDMS are easily introduced. Up to that point, the interconnections were still conductive. No clear conclusion about the eventual lifetime of the interconnections can thus be taken out of the mentioned numbers; they pose a minimum borderline for the reliability.

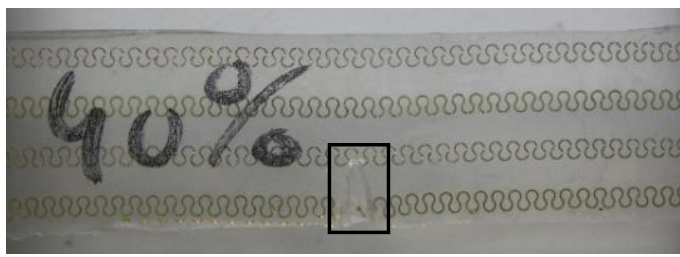

Figure 9: Tearing of PDMS when subjected to a cyclic uni-axial strain of $40 \%$; picture was taken at a strain of approximately $20 \%$.

At the lower strains, the above-mentioned problem did not occur. For the reliability of interconnections subjected to $20 \%$ strain, a trend as before was noticed. The tracks supported with $100 \mu \mathrm{m}$ wide polyimide stopped conducting at about 44'900 cycles while the other tracks were conducting for over 130 '000 cycles. When strains of $10 \%$ were applied, it was found that all of the electrical interconnections kept conducting for over 500'000 cycles. Last two tests were eventually stopped with the interconnections still being conductive and with a hardly measurable resistance change.

\section{Conclusions}

A new thin-film technology is presented for the fabrication of stretchable electrical interconnections. Key features are its finer pitch, possible use in biomedical applications, minimal variation in resistance when subjected to strain and enhanced reliability. Cyclic mechanical loading of test structures fabricated in this technology was performed, and illustrated the influence of polyimide support width on reliability. Fabricated tracks with a $100 \mu \mathrm{m}$ wider polyimide support have a much higher lifetime; a lifetime over 130'000 and 500'000 cycles has been shown at strains of respectively $20 \%$ and $10 \%$. A more elaborate reliability testing and failure analysis of the technology will be performed in the future.

\section{Acknowledgments}

This research was performed in the context of an IMEC PhD grant.

\section{References}

1. Graz, I.M., Cotton, D.P.J., Lacour, S.P., "Extended Cyclic Uniaxial Loading of Stretchable Gold ThinFilms on Elastomeric Substrates," Applied Physics Letters, Vol. 94, No. 7 (2009), pp. 071902.

2. Gonzalez, M., Axisa, F., Vanden Bulcke, M., Brosteaux, D., Vandevelde, B., Vanfleteren, J., "Design of Metal Interconnects for Stretchable Electronic Circuits," Microelectronics Reliability, Vol. 48, No. 6 (2008), pp. 825-832.

3. Axisa, F., Bossuyt, F., Vervust, T., Vanfleteren, J., "Laser Based Fast Prototyping Methodology of Producing Stretchable and Conformable Electronic Systems," $2^{\text {nd }} \quad$ Electronics System-Integration Technology Conference, 2008, pp. 1387-1390.

4. "PI-2600 product bulletin," HD MicroSystems, Revised September 2009 